\title{
Single-shot method for measuring femtosecond bunch length in linac-based free-electron lasers
}

\author{
Z. Huang, K. Bane, Y. Ding, and P. Emma \\ SLAC National Accelerator Laboratory, Menlo Park, California 94025, USA
}

(Received 5 June 2010; published 7 September 2010)

\begin{abstract}
There is growing interest in the generation and characterization of femtosecond and subfemtosecond pulses from linac-based free-electron lasers (FELs). In this report, following the method of Ricci and Smith [Phys. Rev. ST Accel. Beams 3, 032801 (2000)], we investigate the measurement of the longitudinal bunch profile of an ultrashort electron bunch produced by these FELs. We show that this method can be applied in a straightforward manner at x-ray FEL facilities such as the Linac Coherent Light Source by slightly adjusting the second bunch compressor followed by running the bunch on an rf zero-crossing phase of the final linac. We find that the linac wakefield strongly perturbs the measurement, and through analysis show that it can be compensated in a simple way. We demonstrate the effectiveness of this method and wakefield compensation through numerical simulations, including effects of coherent synchrotron radiation and longitudinal space charge. When used in conjunction with a high-resolution electron spectrometer, this method potentially reveals the temporal profile of the electron beam down to the femtosecond and subfemotsecond scale.
\end{abstract}

DOI: 10.1103/PhysRevSTAB.13.092801

PACS numbers: 29.27.Fh, 29.27.Bd, 41.60.Cr

\section{INTRODUCTION}

The advent of $\mathrm{x}$-ray free-electron lasers (FELs) is expected to revolutionize the ultrafast $x$-ray sciences. The realization of the Linac Coherent Light Source (LCLS) [1], with its capability of generating femtosecond electron and $\mathrm{x}$-ray pulses [2], opens up vast opportunities for studying atoms and molecules on an unprecedented time scale. FEL configurations with very low beam charges have been proposed that promise even shorter x-ray pulses, down to the attosecond scale $[3,4]$. However, tremendous challenges remain in the measurement and control of these ultrashort pulses with subfemtosecond or even femtosecond precision.

The measurement of the length of ultrashort bunches is an important but challenging topic. Techniques exist for measuring the coherent radiation spectrum of a short bunch in order to reconstruct its temporal profile (see Ref. [5] for a recent excellent example). Information about the bunch length can also be obtained from the statistical fluctuation of the incoherent radiation intensity [6,7]. The more direct measurement methods include rf zero phasing that introduces an energy-to-time correlation [8] and the use of a transverse deflecting cavity that introduces a transverseto-time correlation [9]. Measurement of the correlated coordinates yields information about the bunch length. The resolution of these different techniques is machine specific, but typically limited to the 100 -fs to 10 -fs levels (see, e.g., Refs. $[8,10,11])$. In particular, the compressed low-charge bunches in the LCLS, which are expected to be less than $10 \mathrm{fs}$ in duration, are too short to be measured currently by these standard techniques. In principle, the resolution of the transverse deflecting method can be improved by increasing the deflecting voltage and $\mathrm{rf}$ frequency of the transverse cavity. Recently, a novel longitudinal-to-transverse mapping method using an $\mathrm{x}$-band deflector has been proposed to further improve the temporal resolution to below the 1-fs level [12].

In this paper, we discuss a simple, single-shot method for measuring the longitudinal bunch profile of ultrashort electron bunches, which uses a chicane followed by an $\mathrm{rf}$ linac. The technique was first suggested in Ref. [13] and developed in Refs. $[14,15]$ to directly observe the longitudinal profile of an FEL microbunched electron beam for a relatively low-energy superconducting linac. In this paper, we show that this method can easily be adapted to linac-based FELs such as the LCLS; one needs only to (1) slightly adjust the final bunch compressor strength and (2) run the beam on the zero-crossing phase in the linac that follows. This method is insensitive to the initial beam energy spread or chirp and hence is more effective than rf zero phasing for very short bunches. It normally requires no extra hardware (e.g., a high-frequency deflecting cavity) in a linac-based FEL. The effect of the wakefield in the linac must be included in the compressor adjustment in order for the final energy spread to correspond directly to the bunch length that we are trying to measure. We analytically derive the setup for this measurement technique and demonstrate its feasibility with simulations of the LCLS in low-charge mode. When used in conjunction with a highresolution spectrometer, this method potentially reveals the temporal profile of the electron beam down to the femtosecond and subfemotsecond scale.

This paper is organized as follows: we begin with a review of the measurement technique and discuss how it can be set up in an x-ray FEL such as the LCLS. In Sec. III, we analytically treat the wakefield effect for a short bunch and show how it can be taken into account in 
TABLE I. LCLS low-charge bunch and linac parameters used in this study.

\begin{tabular}{lcccc}
\hline \hline Parameter & Symbol & Nominal & Measurement & Unit \\
\hline Bunch charge & $e N$ & 20 & & $\mathrm{pC}$ \\
Bunch length in L2 (rms) & $\sigma_{z 2}$ & 38 & $\mu \mathrm{m}$ \\
L2 rf phase & $\phi_{2}$ & -31 & \\
BC2 energy & $E_{2}$ & 4.3 & & $\mathrm{deg}$ \\
BC2 strength & $R_{56}$ & -24.7 & $-32.78^{\mathrm{a}}\left(-31.88^{\mathrm{b}}\right)$ & $\mathrm{GeV}$ \\
Bunch length in L3 (rms) & & $\sigma_{z 3} \sim 1$ & $\bar{\sigma}_{z 3} \sim 10$ & $\mu \mathrm{m}$ \\
L3 rf phase & $\phi_{3}$ & 0 & -90 & $\mathrm{deg}$ \\
L3 length & $L_{3}$ & 552.9 & & $\mathrm{~m}$ \\
Maximum accelerating voltage & $V_{3}$ & 10 & $\mathrm{GV}$ \\
\hline \hline
\end{tabular}

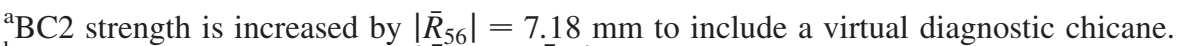

${ }^{\mathrm{b}} \mathrm{BC} 2$ strength is increased by $\left|\bar{R}_{56}+\Delta \bar{R}_{56}\right|=8.08 \mathrm{~mm}$ to correct for the L3 wakefield.

the measurement. In Sec. IV, we perform numerical simulations applied to the LCLS low-charge beam in order to demonstrate the effectiveness of the method and of the wakefield compensation. Finally, in Sec. V, we discuss the resolution limit and possible applications of this method to other x-ray FEL facilities. In Table I we present selected parameters for the LCLS that will be used later in this report for the simulations.

\section{APPLICATION OF THE METHOD}

In a typical x-ray FEL such as the LCLS, after the electron bunch is compressed in the final bunch compressor (that we call BC2 at the electron energy $E_{2}=\gamma_{2} m c^{2}$ ), there is a final linac section (that we call L3) to accelerate the beam to the desired final energy. In addition to the nominal machine configuration, we add a diagnostic chicane right after $\mathrm{BC} 2$ and run the beam in the $\mathrm{L} 3$ linac on the rf zero-crossing phase (where there is no net acceleration). We sketch the setup in Fig. 1. Nominal setup parameters are given in black type, those changed for measurement mode are in red and have an overbar. In this paper, we choose a longitudinal coordinate system such that the head of the bunch is at $z<0$. A chicane has $R_{56}<0$ with this convention.

Suppose the additional chicane strength is $\bar{R}_{56}$, and the rf-induced linear chirp in L3 is

$$
h_{3}= \pm \frac{2 \pi}{\lambda_{\mathrm{rf}}} \frac{e V_{3}}{E_{2}}
$$

where $\lambda_{\mathrm{rf}}$ is the rf wavelength, $V_{3}$ the maximum accelerating voltage of L3, and \pm refers to the two rf zero-crossing phases. Then longitudinal phase space is transformed between the $\mathrm{BC} 2$ end and the $\mathrm{L} 3$ end according to

$$
\begin{aligned}
\left(\begin{array}{l}
\bar{z}_{3} \\
\bar{\delta}_{3}
\end{array}\right) & =\left(\begin{array}{cc}
1 & 0 \\
h_{3} & 1
\end{array}\right)\left(\begin{array}{cc}
1 & \bar{R}_{56} \\
0 & 1
\end{array}\right)\left(\begin{array}{l}
z_{3} \\
\delta_{2}
\end{array}\right) \\
& =\left(\begin{array}{cc}
1 & \bar{R}_{56} \\
h_{3} & 1+h_{3} \bar{R}_{56}
\end{array}\right)\left(\begin{array}{l}
z_{3} \\
\delta_{2}
\end{array}\right),
\end{aligned}
$$

where $z_{3}$ and $\bar{z}_{3}$ are the longitudinal bunch coordinates after $\mathrm{BC} 2$ and the diagnostic chicane, and $\delta_{2}$ and $\bar{\delta}_{3}$ are the relative energy coordinates at the end of L2 and L3, respectively (see Fig. 1). To have a one-to-one correspondence between $z_{3}$ and the final energy coordinate $\bar{\delta}_{3}=$ $h_{3} z_{3}+\left(1+h_{3} \bar{R}_{56}\right) \delta_{2}$ requires that $[13,14]$

$$
1+h_{3} \bar{R}_{56}=0 .
$$

Hence, the final energy coordinate is independent of the initial energy coordinate to first order, and we have

$$
z_{3}=\frac{\bar{\delta}_{3}}{h_{3}}, \quad \text { and } \quad \sigma_{z 3}=\frac{\bar{\sigma}_{\delta 3}}{\left|h_{3}\right|} .
$$

Thus, the final energy profile of the beam is a scaled image of its temporal profile after $\mathrm{BC} 2$, and an energy spectrum measurement yields the bunch profile. In a typical x-ray FEL, the L3 accelerating voltage can be larger than the $\mathrm{BC} 2$ energy by more than a factor of 2 . For the LCLS S-band accelerators with $\lambda_{\mathrm{rf}}=10.5 \mathrm{~cm}$, this yields

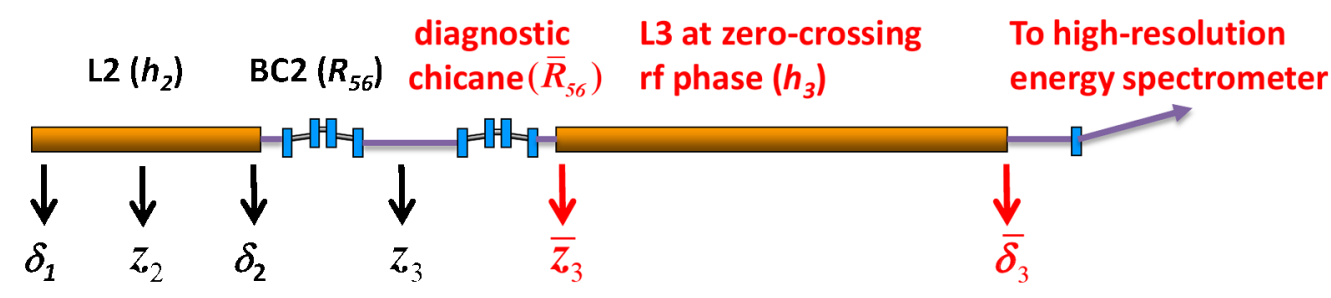

FIG. 1. Schematic setup for short-bunch measurement using a chicane and an rf linac. The arrows give the locations at which the various energy and position symbols apply. In red are parameters that are changed for the purpose of the bunch length measurement. Note that the diagnostic chicane can actually be part of $\mathrm{BC} 2$, with its total strength changed to $R_{56}+\bar{R}_{56}$. 
$\left|h_{3}\right| \geq 100 \mathrm{~m}^{-1}$ for a beam running on the rf zero-crossing phase $\left(h_{3}>0\right.$ for $\left.\bar{R}_{56}<0\right)$. In order to measure an ultrashort electron bunch with $\sigma_{z 3}=1 \mu \mathrm{m}$ (e.g., a possible value in the LCLS low-charge mode after BC2 [2]), the final energy spread after this transformation becomes $\bar{\sigma}_{\delta 3}=\left|h_{3}\right| \sigma_{z 3} \sim 1 \times 10^{-4}$, requiring a high-resolution spectrometer to resolve. In comparison, the rf zero-phasing technique [8] requires that the rf-induced energy spread $\left|h_{3}\right| \sigma_{z 3}$ be larger than the intrinsic beam energy spread $\sigma_{\delta 2}$, a condition that cannot be satisfied for $\sigma_{z 3} \leq 10 \mu \mathrm{m}$ since typically we have $\sigma_{\delta 2} \geq 1 \times 10^{-3}$. The method described here is more sensitive than the zero-phasing technique because the use of the additional chicane results in a longer bunch in the zero-crossing linac section.

We note that the addition of a diagnostic chicane is only conceptual; one can simply increase the strength of $\mathrm{BC} 2$ to perform the measurement. It is also possible to reduce the BC2 $R_{56}$ strength by $\left|\bar{R}_{56}\right|$ instead of increasing it, equivalent to a diagnostic dispersion section with $\bar{R}_{56}>0$. In this case, the L3 phase needs to be set at the other zero-crossing phase (where $h_{3}<0$ ), so that the relation $h_{3} \bar{R}_{56}=-1 \mathrm{can}$ still be satisfied.

The method described here is attractive because it does not require additional hardware beyond what already exists in a linac-based FEL. (We assume, however, that a highresolution spectrometer is available at the end of the linac.) The method also provides single-shot bunch length and shape measurement capabilities. For x-ray FELs driven by higher-frequency rf linacs (C-band or X-band), the final chirp amplitude $\left|h_{3}\right|$ would be increased by a factor of 2 or 4 , which would further enhance the temporal resolution of this method. An important effect that has not been included in the calculations so far is that of the linac wakefield in L3, an effect that will be addressed in the following section.

\section{WAKEFIELD COMPENSATION}

The longitudinal wakefield of the linac L3 induces an additional energy spread in the electron bunch. For L3, let $L$ be the total length, $a$ the average iris radius of the accelerating structure, $s_{0}$ the characteristic length of the wakefield [16], and $\bar{\sigma}_{z 3}$ the rms bunch length. Note that $\bar{\sigma}_{z 3}$, the rms bunch length after the diagnostic chicane in measurement mode, in general will be very different than $\sigma_{z 3}$, the rms bunch length we are trying to measure (see Fig. 1). The wake-induced relative energy change at bunch length coordinate $\bar{z}_{3}$ is (see, e.g., Ref. [16])

$$
\delta_{w}\left(\bar{z}_{3}\right)=-\frac{e^{2} L}{\gamma_{2} m c^{2}} \int_{0}^{\infty} W(z) n\left(\bar{z}_{3}-z\right) d z,
$$

where $W(z)$ is the point charge wake function, $c$ the speed of light, and $n(z)$ the longitudinal bunch distribution with normalization $\int_{-\infty}^{\infty} n(z) d z=N(N$ is the total number of electrons in the bunch). Under the condition

$$
\frac{a^{2}}{2 L} \ll \bar{\sigma}_{z 3} \ll s_{0},
$$

we can use the limiting value of the steady-state wake for periodic structures:

$$
W\left(0^{+}\right)=\frac{Z_{0} c}{\pi a^{2}} .
$$

Here $Z_{0}=377 \Omega$. The first inequality says that the length of structure $L$ is much larger than the catch-up distance $a^{2} /\left(2 \bar{\sigma}_{z 3}\right)$, and the transient behavior of the wake can be ignored. Measurement of wakefield effects for very short bunches in the SLAC linac is in good agreement with the analytical theory used here [17].

Inserting Eq. (7) into Eq. (5), we obtain

$$
\delta_{w}\left(\bar{z}_{3}\right)=-\frac{4 r_{e} L}{\gamma_{2} a^{2}} \int_{0}^{\infty} n\left(\bar{z}_{3}-z\right) d z,
$$

with the classical electron radius $r_{e}=2.82 \times 10^{-15} \mathrm{~m}$. The average energy change per electron is

$$
\bar{\delta}_{w}=\frac{1}{N} \int_{-\infty}^{\infty} \delta_{w}\left(\bar{z}_{3}\right) n\left(\bar{z}_{3}\right) d \bar{z}_{3}=-\frac{2 N r_{e} L}{\gamma_{2} a^{2}},
$$

and the wake-induced rms energy spread in the bunch is given by

$$
\sigma_{\delta_{w}}=\left[\frac{1}{N} \int_{-\infty}^{\infty}\left[\delta_{w}\left(\bar{z}_{3}\right)-\bar{\delta}_{w}\right]^{2} n\left(\bar{z}_{3}\right) d \bar{z}_{3}\right]^{1 / 2}=\frac{2}{\sqrt{3}} \frac{N r_{e} L}{\gamma_{2} a^{2}} .
$$

We emphasize that Eqs. (9) and (10), which apply for a very short bunch, are independent of bunch length and shape. For the LCLS L3, we have $a=11.63 \mathrm{~mm}$, $L=553 \mathrm{~m}$ from Table I, $s_{0}=1.5 \mathrm{~mm}$ for the SLAC S-band structure [16], and the typical bunch length after the diagnostic chicane is $\bar{\sigma}_{z 3} \sim 10 \mu \mathrm{m} \ll s_{0}$ for the 20 $\mathrm{pC}$-charge bunch (see next section for details). The catchup distance for the linac wakefield is then $a^{2} /\left(2 \bar{\sigma}_{z 3}\right) \sim$ $7 \mathrm{~m} \ll L=553 \mathrm{~m}$, and hence the steady-state wake is a good approximation here. Thus, both inequalities in Eq. (6) are well satisfied for the parameters of interest here. Equation (10) predicts that the wake-induced energy spread is $2 \times 10^{-4}$ at $4.3 \mathrm{GeV}$ (for $N=1.25 \times 10^{8}$ ), comparable to the size of energy spread we need to measure in order to find the bunch length $\sigma_{z 3}$. However, the wakeinduced energy spread is correlated with the longitudinal bunch coordinate and can be compensated by a proper adjustment of compression setting, as we will now show.

Following Eq. (2) with the requirement $1+h_{3} \bar{R}_{56}=0$, we add the wake-induced energy change to the final energy coordinate as

$$
\bar{\delta}_{3}=h_{3} z_{3}+\delta_{w}\left(\bar{z}_{3}\right) .
$$

We can also relate

$$
z_{3}=\left(1+h_{2} R_{56}\right) z_{2}+R_{56} \delta_{1}, \quad \delta_{2}=h_{2} z_{2}+\delta_{1},
$$


where $z_{2}$ and $\delta_{1}=\Delta E_{2} / E_{2}$ are the initial bunch length coordinate and relative energy deviation at the beginning of the L2 linac section, and $h_{2}$ is the linear chirp induced by L2 (see Fig. 1). When the L2 phase is set to $\phi_{2}$ (here phase is defined with respect to the rf crest), and the L2 energy gain is much larger than the initial beam energy, we have

$$
h_{2} \approx-\left(\frac{2 \pi}{\lambda_{\mathrm{rf}}}\right) \tan \phi_{2} \text {. }
$$

If we now shift the L2 phase slightly from $\phi_{2}$ to $\phi_{2}^{s}$, but without significantly affecting the $\mathrm{BC} 2$ energy, then the longitudinal phase space coordinates after $\mathrm{BC} 2$ become

$$
z_{3}^{s}=\left(1+h_{2}^{s} R_{56}\right) z_{2}+R_{56} \delta_{1}, \quad \delta_{2}^{s}=h_{2}^{s} z_{2}+\delta_{1},
$$

where $h_{2}^{s}$ is the L2 chirp corresponding to phase $\phi_{2}^{s}$. With help from Eqs. (11), (12), and (14), the corresponding energy coordinate at the end of L3 becomes

$$
\begin{aligned}
\bar{\delta}_{3}^{s}= & h_{3} z_{3}^{s}+\delta_{w}\left(\bar{z}_{3}^{s}\right) \\
= & h_{3}\left[\left(1+h_{2}^{s} R_{56}\right) z_{2}+R_{56} \delta_{1}\right]+\delta_{w}\left(\bar{z}_{3}^{s}\right) \\
= & h_{3}\left[\left(1+h_{2} R_{56}\right) z_{2}+R_{56} \delta_{1}\right]+h_{3} \Delta h_{2} R_{56} z_{2} \\
& +\delta_{w}\left(\bar{z}_{3}^{s}\right) \\
= & h_{3} z_{3}+h_{3} \Delta h_{2} R_{56} z_{2}+\delta_{w}\left(\bar{z}_{3}^{s}\right),
\end{aligned}
$$

where $\Delta h_{2}=h_{2}^{s}-h_{2}$ is the shift in L2 chirp, which can be set so that the second term of the last expression cancels the wake-induced energy chirp $\delta_{w}$.

For a uniform longitudinal bunch profile, we have $n(z)=N /\left(2 \sqrt{3} \sigma_{z}\right)$ for $|z| \leq \sqrt{3} \sigma_{z}$ and $n(z)=0$ for $|z|>$ $\sqrt{3} \sigma_{z}$, and Eq. (8) yields a linear wake-induced energy change along the bunch coordinate

$$
\delta_{w}\left(\bar{z}_{3}^{s}\right)=-\frac{2 N r_{e} L}{\gamma_{2} a^{2}}\left(1+\frac{\bar{z}_{3}^{s}}{\sqrt{3} \bar{\sigma}_{z 3}^{s}}\right) \text { for }\left|\bar{z}_{3}^{s}\right|<\sqrt{3} \bar{\sigma}_{z 3}^{s} .
$$

From Eqs. (2) and (14), we also have

$$
\bar{z}_{3}^{s}=z_{3}^{s}+\bar{R}_{56} \delta_{2}^{s} \approx\left[1+h_{2}^{s}\left(R_{56}+\bar{R}_{56}\right)\right] z_{2},
$$

if we neglect the small energy term $\delta_{1}$ in Eq. (14). With the same approximation, we have $\bar{\sigma}_{z 3}^{s} \approx \mid 1+h_{2}^{s}\left(R_{56}+\right.$ $\left.\bar{R}_{56}\right) \mid \sigma_{z 2}$, where $\sigma_{z 2}$ is the rms bunch length in L2. Therefore, Eq. (16) becomes

$$
\delta_{w} \approx-\frac{2 N r_{e} L}{\gamma_{2} a^{2}}\left(1-\frac{z_{2}}{\sqrt{3} \sigma_{z 2}}\right),
$$

where we have changed the sign in front of the second term because $1+h_{2}^{s}\left(R_{56}+\bar{R}_{56}\right)<0$. The first term on the right side of Eq. (18) is a constant (the average wake energy loss); the second term is proportional to $z_{2}$ and can be made to cancel the term $h_{3} \Delta h_{2} R_{56} z_{2}$ in Eq. (15). The condition for cancellation is

$$
\Delta h_{2}=-\frac{2 N r_{e} L}{\sqrt{3} \gamma_{2} a^{2}\left|h_{3}\right| R_{56} \sigma_{z_{2}}}=-\frac{\sigma_{\delta_{w}}}{\left|h_{3}\right| R_{56} \sigma_{z_{2}}} .
$$

Here the last equality is obtained using Eq. (10), and the absolute value of $h_{3}$ appears in order to include the other zero-crossing possibility (with $h_{3}<0$ but with the position of bunch head and tail interchanged). Under the condition of Eq. (19), we arrive back at

$$
z_{3}=\frac{\bar{\delta}_{3}^{s}}{h_{3}}, \quad \text { and } \quad \sigma_{z 3}=\frac{\bar{\sigma}_{\delta 3}^{s}}{\left|h_{3}\right|} .
$$

Thus, the bunch length change introduced by the additional chirp in L2 induces an additional rf energy chirp in L3 that cancels the wakefield contribution. Although Eq. (19) is derived for a uniform current profile, the wake compensation works well for a Gaussian current distribution (see the Appendix). Section IV will demonstrate it also works for actual current distributions produced by simulations.

Combining Eqs. (13) and (19), we find the correct L2 phase shift to compensate the wake is

$$
\begin{aligned}
\Delta \phi_{2} & =\phi_{2}^{s}-\phi_{2} \approx-\frac{\lambda_{\mathrm{rf}} \cos ^{2}\left(\phi_{2}\right) \Delta h_{2}}{2 \pi} \\
& \approx \sqrt{\frac{8 \pi}{3}} \frac{I_{2}}{I_{A}} \frac{\lambda_{\mathrm{rf}} \cos ^{2}\left(\phi_{2}\right) L}{2 \pi \gamma_{2} a^{2} R_{56}\left|h_{3}\right|},
\end{aligned}
$$

where $I_{2} / I_{A}=r_{e} N /\left(\sqrt{2 \pi} \sigma_{z 2}\right)$ is the peak current in L2 (for a Gaussian bunch) in terms of the Alfvén current $I_{A}=17045 \mathrm{~A}$. Thus, the shift in L2 phase is independent of the bunch length $\sigma_{z 3}$ that we are trying to measure.

An even simpler way to compensate the wakefield effect is to change $\bar{R}_{56}$ instead of $\phi_{2}$ since it can be a part of compressor setting adjustment. The required shift in $\bar{R}_{56}$ can be similarly calculated as [again assuming $\delta_{1}$ in Eq. (14) is small]

$$
\Delta \bar{R}_{56}=\bar{R}_{56}^{s}-\bar{R}_{56}=\frac{-\sigma_{\delta_{w}}}{h_{2}\left|h_{3}\right| \sigma_{z_{2}}} \approx \sqrt{\frac{8 \pi}{3}} \frac{I_{2}}{I_{A}} \frac{R_{56} L}{\gamma_{2} a^{2}\left|h_{3}\right|},
$$

where we have used the $\mathrm{BC} 2$ compression condition $h_{2} R_{56} \approx-1$ to simplify the last expression. Therefore, in order to measure the compressed bunch length after BC2 under the nominal operating $R_{56}$, we can increase the $\mathrm{BC} 2$ strength by $\left|\bar{R}_{56}+\Delta \bar{R}_{56}\right|$ according to Eqs. (3) and (22), and run the beam in L3 at an rf zero-crossing phase $\left(-90^{\circ}\right)$. The measured energy profile at the end of L3 divided by $\left|h_{3}\right|$ gives the bunch shape (or its mirror image) after $\mathrm{BC} 2$ at the original $R_{56}$ setting. Alternatively, we can decrease the BC2 strength by $\left|\bar{R}_{56}\right|+\Delta \bar{R}_{56}$, and run the beam in L3 at the other rf zero-crossing phase $\left(+90^{\circ}\right)$ to obtain the same results.

\section{SIMULATION STUDIES}

\section{A. LITRACK simulations}

We first demonstrate the proposed method with LITRACK simulations. LITRACK [18] is a longitudinal phase space 
tracking code that includes sinusoidal rf acceleration, bunch compression dynamics up to third order, and longitudinal wakefield effects. We use parameters that are representative of the LCLS operation in low-charge mode (see Table I). In the LCLS, between the rf gun and the undulator, there are linacs and bunch compressors in the sequence: Linac-0, Linac-1, BC1, Linac-2, BC2, Linac-3. The rms bunch length in Linac-0 is $\sim 270 \mu \mathrm{m}$, and the rms slice energy spread (in the central part of the bunch) is $\sim 0.5 \mathrm{keV}$. The Linac- 1 and $\mathrm{BC} 1$ parameters are the same as in Table 1 of Ref. [10] and are not given here. The maximum total $\mathrm{rf}$ accelerating voltage in L3 (Linac-3) is $\sim 10 \mathrm{GV}$ (see Table I), which yields $\left|h_{3}\right| \approx 139 \mathrm{~m}^{-1}$ according to Eq. (1). To simulate our measurement technique in the LCLS beam line without adding a diagnostic chicane, we increase the BC2 strength by $\left|\bar{R}_{56}\right|=1 /\left|h_{3}\right| \approx$ $7.18 \mathrm{~mm}$ (i.e., changing $R_{56}$ from its nominal value $-24.7 \mathrm{~mm}$ to $-31.88 \mathrm{~mm}$ ). The bunch is overcompressed with the additional chicane strength to a final rms bunch length $\bar{\sigma}_{z 3} \sim 10 \mu \mathrm{m}$. It then traverses L 3 at the $-90^{\circ}$ zerocrossing phase to induce a positive chirp $h_{3} \approx+139 \mathrm{~m}^{-1}$ (so that $h_{3} \bar{R}_{56}=-1$ ). An example of the energy profile after L3 is shown in Fig. 2 (green curve). In this example, the L2 phase is set at $\phi_{2}=-31^{\circ}$, and the L3 wakefield is turned off in the simulation. The current profile after the nominal $\mathrm{BC} 2$ setting (with $R_{56}=-24.7 \mathrm{~mm}$ ) is also shown in Fig. 2 for comparison (blue curve). The two curves almost overlap each other.

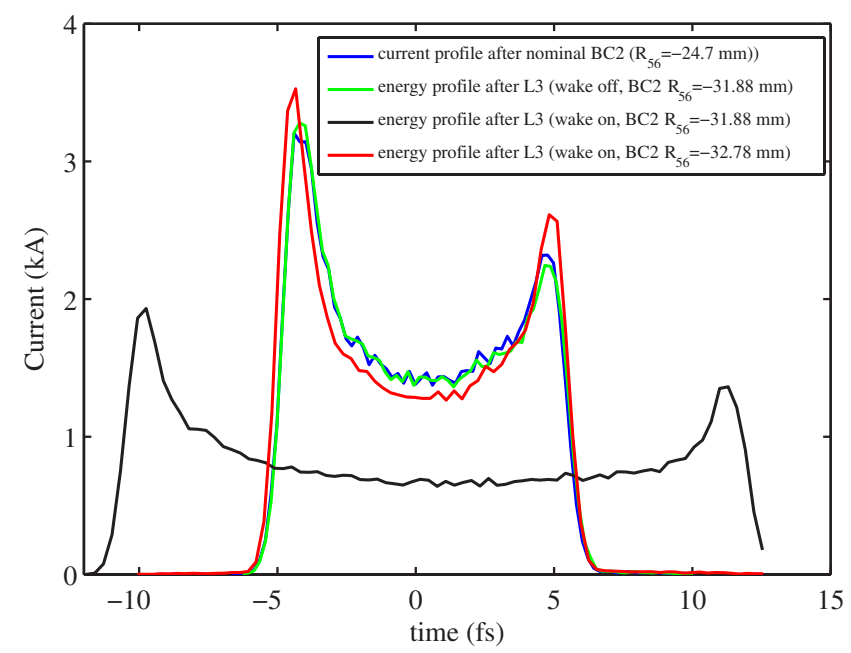

FIG. 2. LITRACK simulations showing current profile of the LCLS 20-pC, ultrashort bunch after the nominal BC2 setting (with $R_{56}=-24.7 \mathrm{~mm}$ ) (blue curve), energy profile after increasing BC2 strength (by $\left|\bar{R}_{56}\right|=7.18 \mathrm{~mm}$ ) and traversing L3 at the $-90^{\circ}$ zero-crossing phase (green curve for L3 wake off and black curve for L3 wake on), and energy profile after increasing $\mathrm{BC} 2$ strength (by $\left|\bar{R}_{56}+\Delta \bar{R}_{56}\right|=8.08 \mathrm{~mm}$ ) and traversing L3 at the $-90^{\circ}$ zero-crossing phase (L3 wake on, red curve). The energy coordinate is divided by $h_{3}$ according to Eq. (4) and is converted to time by speed of light. L2 phase $\phi_{2}=-31^{\circ}$ here.
When the L3 wakefield is turned on in LITRACK, the energy profile (black curve in Fig. 2) is significantly distorted as compared to the current profile we try to measure. Adjusting the diagnostic chicane strength $\bar{R}_{56}$ according to Eq. (22) will compensate for the wakefield effect. Since the peak current in L2 is $I_{2} \approx 60 \mathrm{~A}$ from LITRACK, we compute $\Delta \bar{R}_{56} \approx-0.9 \mathrm{~mm}$. Thus, we can increase $\mathrm{BC} 2$ strength by $\left|\bar{R}_{56}+\Delta \bar{R}_{56}\right|=8.08 \mathrm{~mm}$ to $32.78 \mathrm{~mm}$. After this adjustment, the energy profile after L3 is shown as the red curve in Fig. 2, which agrees well with the current profile after the nominal BC2 setting (blue curve). The small discrepancies can be attributed to the nonlinearity of the wakefield effect not easily compensated for. We now vary the L2 phase $\phi_{2}$, thus changing the bunch length after $\mathrm{BC} 2$, and see how well our measurement technique continues to work. Figure 3 shows the simulated rms bunch length in fs (i.e., $\sigma_{z 3} / c$ ) after the nominal $\mathrm{BC} 2$ (blue diamonds), as well as the "measured" rms bunch length $\left(\bar{\sigma}_{\delta 3} / c /\left|h_{3}\right|\right)$ for comparison (red circles). The rms bunch length and energy spread are obtained by making a 5\% area cut in the $z$-and $\delta$-distribution tails in order to emphasize the bunch core. We see that the measurement technique and the wakefield compensation work for a wide range of bunch length.

As discussed in Sec. III, one can also compensate for the wakefield effect by shifting $\phi_{2}$ instead of $\bar{R}_{56}$. In this case, the $\mathrm{BC} 2$ strength is only increased by $\bar{R}_{56}$ to include the virtual diagnostic chicane. Figure 4 shows the "real" rms bunch length (blue diamonds), and the measured rms bunch length based on the rms energy spread after L3 (red squares). We see that the measured bunch length does not agree with the real bunch length due to the wakefield effect. However, shifting the measured data back by $1^{\circ}$ in the L2 phase recovers the agreement (green circles).

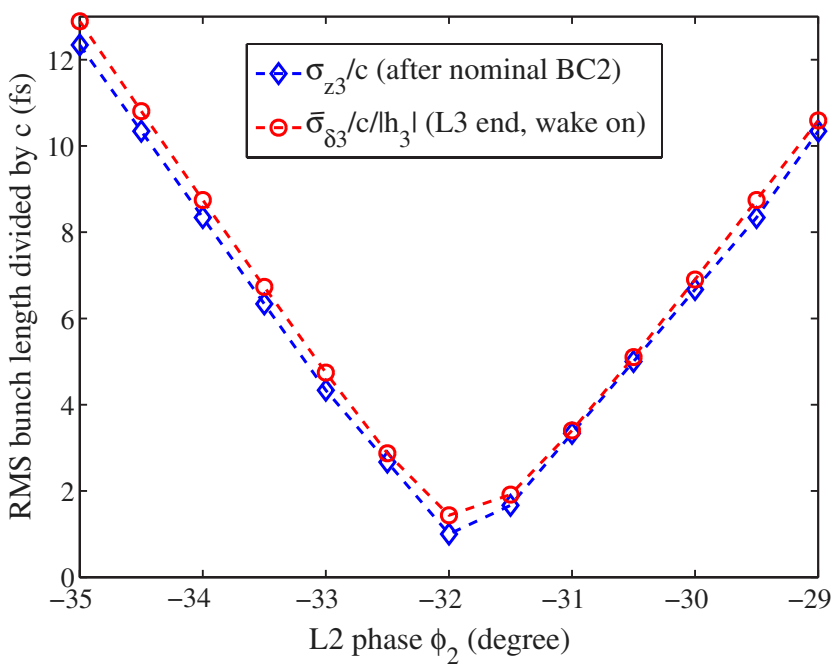

FIG. 3. LITRACK simulations of rms bunch length for the LCLS 20 -pC bunch after BC2 $(5 \%$ area cut in current or energy distributions). The nominal $\mathrm{BC} 2 R_{56}=-24.7 \mathrm{~mm}$, and $R_{56}=$ $-32.78 \mathrm{~mm}$ for the bunch length measurement. 


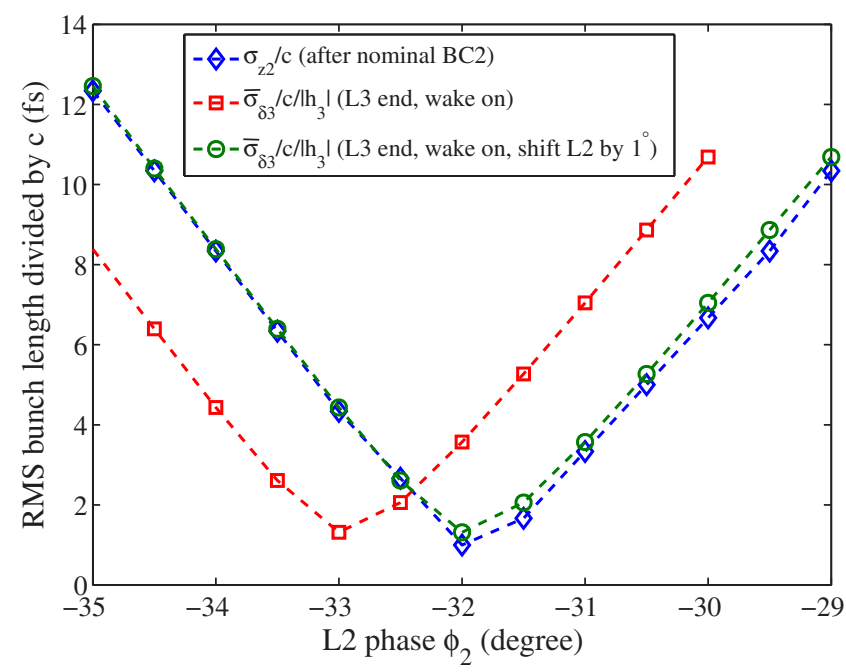

FIG. 4. LITRACK simulations of rms bunch length for the LCLS 20 -pC bunch after BC2 (5\% area cut in current or energy distributions). The nominal $\mathrm{BC} 2 R_{56}=-24.7 \mathrm{~mm}$, and $R_{56}=$ $-31.88 \mathrm{~mm}$ for the bunch length measurement.

Such a phase shift can be predicted from Eq. (21) and can also be found empirically as follows. In the LCLS, a coherent radiation detector after $\mathrm{BC} 2$ is used to monitor the bunch length; it can determine the L2 phase corresponding to full compression to $\pm 0.2^{\circ}$ precision [19]. Thus, the necessary L2 phase shift can be obtained by comparing the energy spread minimum using this measurement technique with the coherent synchrotron radiation (CSR) signal maximum after the nominal $\mathrm{BC} 2$ setting. This can be used as an independent check on wakefield compensation calculation.

We note that the "virtual" diagnostic chicane has a second-order momentum compaction $T_{566} \approx-3 \bar{R}_{56} / 2$ which is not taken into account by Eq. (2). A rough estimate of this contribution to the temporal resolution is $T_{566} \sigma_{\delta 2}^{2}$. For the numerical examples discussed here, $T_{566} \sim 10 \mathrm{~mm}, \sigma_{\delta 2} \sim 1 \times 10^{-3}$, and hence $T_{566} \sigma_{\delta 2}^{2} \sim$ $10 \mathrm{~nm}$ or 30 attosecond and has little effect on the results. Small discrepancies on a few percent level between the measured and the real bunch lengths in Figs. 3 and 4 are due to the nonlinear energy chirp induced by the wakefield of a nonuniform current distribution. The relative level of discrepancies increases near the full compression phase because the bunch length there is determined by the uncorrelated energy spread and higher-order energy-time correlation of the beam. The absolute errors between the measured and the real bunch lengths are everywhere less than $0.4 \mathrm{fs}$.

\section{B. ELEGANT simulations}

We use the six-dimensional ELEGANT tracking code [20] to include the effects of incoherent and coherent synchrotron radiation in bends and longitudinal space charge

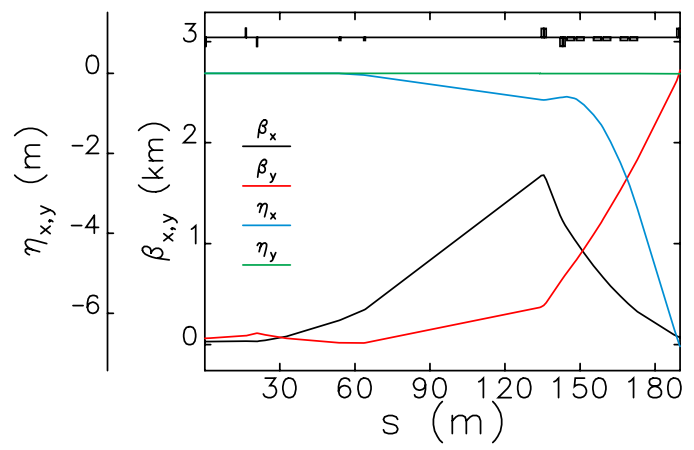

FIG. 5. A-line optics (beta and dispersion) functions from the end of the LCLS linac.

(LSC) in linacs because they may also distort the energy spread measurement. The one-dimensional (1D) algorithm of CSR in ELEGANT was well benchmarked with the LCLS bunch compression measurement recently [10], while the 1D algorithm of LSC used there was compared favorably with 3D space charge codes in Ref. [21]. The LCLS second dogleg spectrometer resolution is $\sim 2 \times 10^{-4}$ [22], and hence is not able to resolve the $1 \mu \mathrm{m}$ rms bunch length that motivates this work. SLAC's end-of-linac to target transfer line (the so-called A-line) has a very large dispersion at the midpoint of a $24.5^{\circ}$ arc [23], and it is planned to deliver the LCLS beams there for high-energy physics detector research and development. Hence, we may use a profile monitor (PR18) located at a high dispersion point in the A-line as a spectrometer. The first 190-m of a modified A-line optics is shown in Fig. 5. The modifications involve changing only the strength of existing quadrupoles in front and inside the A-line in order to reduce the CSR effects in the bends there (see the next paragraph). PR18 is located at $s=188 \mathrm{~m}$ from the beginning of the A-line (see Fig. 5), where the horizontal dispersion $\eta_{x}=-6.39 \mathrm{~m}$, and the beta function $\beta_{x}=99 \mathrm{~m}$. For a typical 20-pC compressed bunch, the normalized emittance at the end of the linac $\gamma_{2} \varepsilon_{x} \leq 0.5 \mu \mathrm{m}$, meaning that the energy resolution at $4.3 \mathrm{GeV}$ is limited by the transverse beam size at the level of $1.2 \times 10^{-5}$. In view of Eq. (4), the temporal resolution is about $90 \mathrm{~nm}$, or $0.3 \mathrm{fs}$. We emphasize that the transverse effects and realistic bunch profiles are all included in the ELEGANT simulations shown here.

Figure 6(a) shows longitudinal phase space after BC2 (with $R_{56}=-24.7 \mathrm{~mm}$ for an undercompressed bunch when the L2 phase is at $\left.\phi_{2}=-31.5^{\circ}\right)$. After increasing the BC2 strength by $\left|\bar{R}_{56}+\Delta R_{56}\right|=8.08 \mathrm{~mm}$ (to $R_{56}=$ $-32.78 \mathrm{~mm}$ ) and running the beam at the $-90^{\circ}$ zerocrossing phase in L3, longitudinal phase space is transformed to Fig. 6(b). Note that we turned off the laser heater in simulations since it is not used in the LCLS low-charge operation. The LSC microbunching is present but is not very strong with such a low-charge beam. As shown in Fig. 6(b), short-wavelength energy modulations due to LSC develop in L3. However, they mainly affect the slice 
(a) Long. phase space after nominal BC2

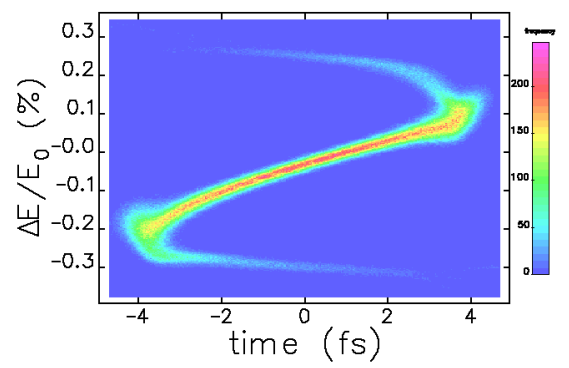

(c) Transverse profile on PR18

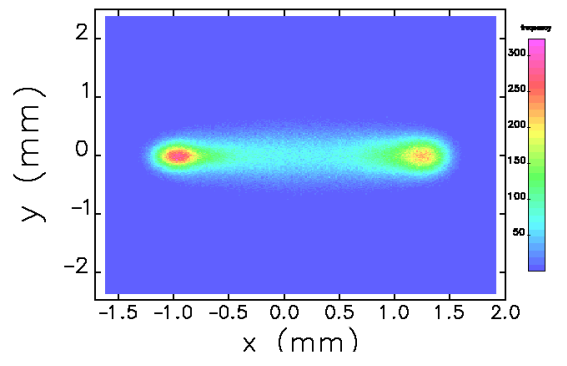

(b) Long. phase space after L3

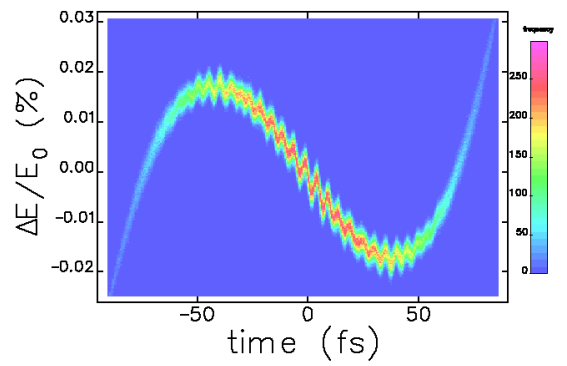

(d) Extracted current profile

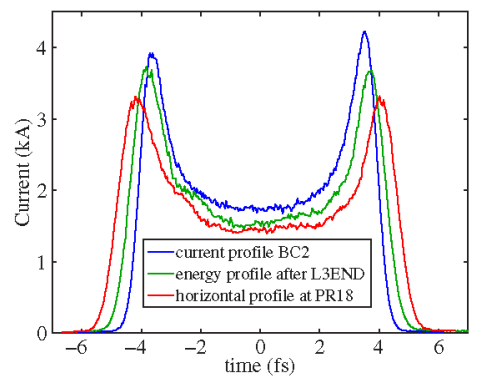

FIG. 6. ELEGANT simulation for an undercompressed 20-pC bunch (L2 phase $\phi_{2}=-31.5^{\circ}$ ), including CSR effects in bends, wakefield, and LSC in linacs.

but not the projected energy spread. The overall energy profile after L3 [green curve in Fig. 6(d)] is not so sensitive to the microbunching instability and can still be used to approximate the current profile [blue curve in Fig. 6(d)]. The beam is then dispersed on PR18 of the A-line to more than $1 \mathrm{~mm}$ in horizontal size [see Fig. 6(c)]. One clearly sees the double-horn current distribution for an undercompressed bunch. The current profile deduced from the horizontal beam profile at PR18 is shown in Fig. 6(d) (red curve). It is slightly wider than the energy and current profiles to be measured (green and blue curves). This small discrepancy is due to the CSR effect in the A-line. The $R_{56}$ of the A-line up to PR18 is $0.24 \mathrm{~m}$ (opposite sign of a chicane), which can compress the bunch with a negative energy chirp and generate CSR in the bends. By modifying the beta functions of the existing A-line to what is shown in
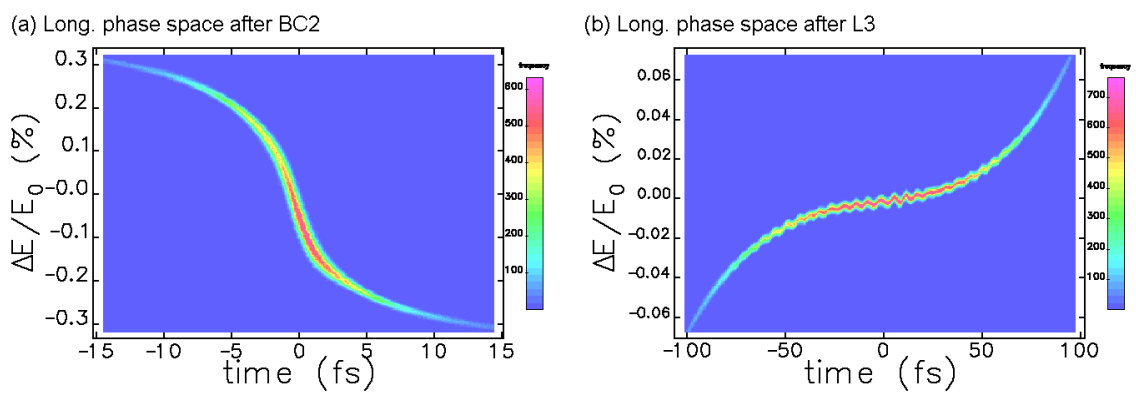

(c) Transverse profile on PR18

(d) Extracted current profile

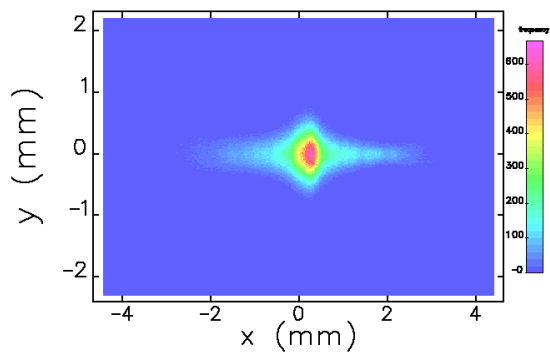

FIG. 7. ELEGANT simulation for an overcompressed bunch (L2 phase $\left.\phi_{2}=-33^{\circ}\right)$, including CSR effects in bends, wakefield, and LSC in linacs. 


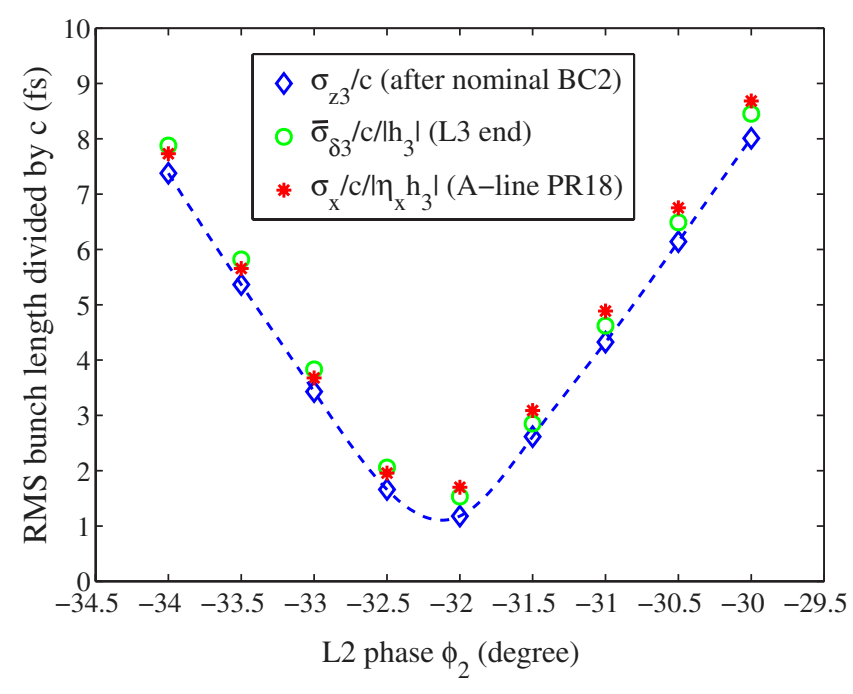

FIG. 8. ELEGANT simulations of rms bunch length for the LCLS 20-pC bunch after BC2 (5\% cut in current, energy, or horizontal distributions, BC2 $R_{56}=-24.7 \mathrm{~mm}$ for operation and $R_{56}=-32.78 \mathrm{~mm}$ for bunch length measurements).

Fig. 5, we introduce correlations in $x-z$ and $x^{\prime}-z$ at locations where the bunch length is small to reduce the CSR effects.

The longitudinal phase space after $\mathrm{BC} 2$ and after $\mathrm{L} 3$, as well as the transverse beam profile on PR18, for an operational overcompressed bunch are shown in Figs. 7(a)-7(c), respectively. The current profile projected on PR18 is shown in Fig. 7(d) (red curve), in good agreement with the simulated energy and current profiles [green and blue curves in Fig. 7(d)]. When turning on and off the secondorder optics in ELEGANT, the simulation results shown in Figs. 6 and 7 were left essentially unchanged.

Finally, Fig. 8 gives the rms bunch lengths as functions of the L2 phase. We see good agreement between the real and measured bunch lengths, with the measured ones $\sim 10 \%$ higher than the real ones in most cases. The discrepancies are attributed to the combination of collective effects: the nonlinear wakefield chirp, CSR and LSC. Simulations done with the opposite sign of $\bar{R}_{56}$ and $+90^{\circ}$ L3 zero-crossing phase yield very similar results. We conclude that a profile monitor in the high-dispersive location of the A-line can resolve the femtosecond electron bunches generated by the LCLS accelerator.

\section{DISCUSSION}

In this paper, we have studied a single-shot method for measuring the longitudinal profile of femtosecond-long bunches in linac-based FELs. The technique requires no additional hardware beyond that already found in normal FEL designs. The linac wakefield needs to be compensated according to Eqs. (21) or (22) (for a very short bunch). In the LCLS, it can also be corrected empirically based on a relative bunch length monitor signal after the final bunch compressor. The temporal resolution of this method is determined by the electron energy spectrometer. Using the LCLS accelerator and the A-line spectrometer, we have shown it is possible to measure the LCLS bunch length and shape with subfemtosecond temporal resolutions. In fact, the measurement of $\sim 1-\mu \mathrm{m}$ bunch length becomes the measurement of $\sim 1$-mm transverse beam size, a relatively easy task. In addition, the higher-order optics has little effect on this measurement technique.

A more important resolution limit is given by collective effects in the accelerator. The linac wakefield can induce a nonlinear energy chirp that may distort the measured energy profile as compared to the current profile. LSC and CSR fields can have similar effects to the measurement technique. Through extensive numerical simulations, we show these effects slightly perturb the LCLS measurement results and yields a systematic error on the order of $0.5 \mathrm{fs}$.

Although only LCLS examples are considered in this paper, we expect that this short-bunch measurement technique is equally applicable to other linac-based FELs. For example, FERMI FEL at Sincrotrone Trieste has an energy spectrometer at the end of its linac with a relative energy resolution $2.5 \times 10^{-5}$ [24], similar to the level discussed here. As mentioned earlier, this method will be more effective for FELs driven by higher-frequency rf linacs (that generates more chirp and energy spread), such as the SPring-8 XFEL [25] and SwissFEL [26] that use C-band linacs, albeit with increased linac wake strength. It may also be applicable to the European XFEL with L-band accelerators [27] since there the final linac is very long, and the wakefield is smaller than in S- and Cband linacs. Further studies are necessary to determine the ultimate resolution of this technique for these x-ray FELs.

\section{ACKNOWLEDGMENTS}

We thank S. Di Mitri, J. Frisch, R. Iverson, Y. Nosochkov, D. Ratner, and D. Xiang for useful discussions. This work was supported by Department of Energy Contract No. DE-AC02-76SF00515.

\section{APPENDIX: WAKEFIELD CHIRP FOR A SHORT, GAUSSIAN BUNCH}

For a nonuniform current distribution in L3, the wakeinduced energy loss is nonlinear with respect to the longitudinal coordinate $\bar{z}_{3}$. The effective linear chirp can be obtained by averaging the instantaneous chirp over the longitudinal density profile as

$$
h_{\mathrm{eff}}=\frac{1}{N} \int_{-\infty}^{\infty} n\left(\bar{z}_{3}\right) \frac{d \delta_{w}}{d \bar{z}_{3}} d \bar{z}_{3} .
$$

For a very short bunch, we can use Eq. (8) to calculate $\delta_{w}$ and arrive at

$$
h_{\mathrm{eff}}=-\frac{4 r_{e} L}{N \gamma_{2} a^{2}} \int_{-\infty}^{\infty} n^{2}\left(\bar{z}_{3}\right) d \bar{z}_{3} .
$$


For a Gaussian bunch in Linac-3, the longitudinal density profile takes the form

$$
n\left(\bar{z}_{3}\right)=\frac{N}{\sqrt{2 \pi} \bar{\sigma}_{z 3}} \exp \left(\frac{-\bar{z}_{3}^{2}}{2 \bar{\sigma}_{z 3}^{2}}\right)
$$

with the rms bunch length $\bar{\sigma}_{z 3}$. Then the effective linear chirp is

$$
h_{\mathrm{eff}}=-\frac{2 N r_{e} L}{\gamma_{2} a^{2} \sqrt{\pi} \bar{\sigma}_{z 3}} .
$$

Comparing the effective linear chirp from a Gaussian bunch to the linear chirp from a flattop bunch [i.e., Eq. (16)], the difference is between $\sqrt{\pi}$ and $\sqrt{3}$ (only $2 \%$ difference). Thus, the wakefield compensation described in Sec. III can be applied to a Gaussian bunch as well.

[1] P. Emma et al., Nat. Photon. 4, 641 (2010).

[2] Y. Ding et al., Phys. Rev. Lett. 102, 254801 (2009).

[3] J. Rosenzweig et al., Nucl. Instrum. Methods Phys. Res., Sect. A 593, 39 (2008).

[4] S. Reiche et al., Nucl. Instrum. Methods Phys. Res., Sect. A 593, 45 (2008).

[5] B. Schmidt et al., in Proceedings of the 11th European Particle Accelerator Conference, Genoa, 2008 (EPS-AG, Genoa, Italy, 2008), p. 130.

[6] M. Zolotorev and G. Stupakov, in Proceedings of the Particle Accelerator Conference, Vancouver, BC, Canada, 1997 (IEEE, New York, 1997), p. 2180.

[7] J. Krzywinski et al., Nucl. Instrum. Methods Phys. Res., Sect. A 401, 429 (1997).

[8] D. X. Wang, G. A. Krafft, and C. K. Sinclair, Phys. Rev. E 57, 2283 (1998).

[9] R. Akre et al., in Proceedings of the Particle Accelerator Conference, Chicago, IL, 2001 (IEEE, New York, 2001), p. 2353.
[10] K. Bane et al., Phys. Rev. ST Accel. Beams 12, 030704 (2009).

[11] M. Rohrs et al., Phys. Rev. ST Accel. Beams 12, 050704 (2009).

[12] D. Xiang and Y. Ding, Report No. SLAC-PUB-14100, 2010.

[13] E. R. Crosson et al., in Micro Bunches Workshop, edited by M. Blum et al., AIP Conf. Proc. No. 367 and No. 397 (AIP, New York, 1996).

[14] K. Ricci and T. Smith, Phys. Rev. ST Accel. Beams 3, 032801 (2000).

[15] K. Ricci, E. R. Crosson, and T. Smith, Nucl. Instrum. Methods Phys. Res., Sect. A 445, 333 (2000).

[16] K. Bane, in Workshop on the Physics and Applications of High Brightness Electron Beams, Erice, Italy, 2005 (World Scientific, New Jersey, 2007); SLAC-PUB11829, 2006.

[17] K. Bane et al., in Proceedings of the 20th Particle Accelerator Conference, Portland, OR, 2003 (IEEE, New York, 2003), p. 3126.

[18] K. Bane and P. Emma, in Proceedings of the 21st Particle Accelerator Conference, Knoxville, 2005 (IEEE, Piscataway, NJ, 2005), p. 4266.

[19] H. Loos et al., in Proceedings of the 2007 Particle Accelerator Conference, Albuquerque, New Mexico (IEEE, Albuquerque, New Mexico, 2007), p. 4189.

[20] M. Borland, ANL/APS LS-287, 2000.

[21] Z. Huang et al., Phys. Rev. ST Accel. Beams 7, 074401 (2004).

[22] Linac Coherent Light Source (LCLS) Conceptual Design Report, SLAC-R-593, 2002.

[23] S. Molloy et al., in Proceedings of the 2007 Particle Accelerator Conference, Albuquerque, New Mexico (Ref. [19]), p. 4201.

[24] Fermi@Elettra conceptual design report, Elettra (2007) [http://www.elettra.trieste.it/FERMI].

[25] SPring-8 Compact SASE Source Conceptual Design Report (2005) [http://www-xfel.spring8.or.jp].

[26] B. D. Patterson et al., New J. Phys. 12, 035012 (2010).

[27] M. Altarelli et al., Report No. DESY 2006-097, 2006. 